\title{
miRNA-27a promotes the proliferation and inhibits apoptosis of human pancreatic cancer cells by Wnt/ß-catenin pathway
}

\author{
ZHIGANG CUI, GENG LIU and DI KONG \\ Department of Oncology, Tianjin Nankai Hospital, Tianjin 300100, P.R. China
}

Received May 3, 2017; Accepted October 9, 2017

DOI: $10.3892 /$ or.2017.6120

\begin{abstract}
A specific expression of miRNA in pancreatic cancer renders it the novel diagnostic marker of pancreatic cancer. Therefore, we investigated how the anticancer effect of miRNA-27a suppressed cell growth and induced apoptosis of human pancreatic cancer cells. We upregulated miRNA-27a expression in PANC-1 cells using miRNA-27a mimic, which demonstrated that induction of cell growth and suppression of apoptosis of human pancreatic cancer cells were observed. However, anti-miRNA-27a inhibited cell growth and apoptosis in pancreatic cancer cells. The downregulation of miRNA-27a suppressed Wnt/ $\beta$-catenin pathway. The inhibition of Wnt/ $\beta$-catenin pathway increased the anticancer effects of anti-miRNA-27a on human pancreatic cancer cells. Taken together, miRNA-27a promotes the proliferation and inhibits apoptosis of human pancreatic cancer cells via $\mathrm{Wnt} / \beta$-catenin pathway.
\end{abstract}

\section{Introduction}

In recent years, the death rate of pancreatic cancer ranks in the top five of malignant cancers in developed countries with its morbidity ascending (1). More than $90 \%$ of pancreatic malignant tumor originates from pancreatic ductal adenocarcinoma which highly concentrates in malignancy and appears mlignant in prognosis (2). Reliable early diagnostic markers and effective therapies are still inadequate. Approximately $60 \%$ of patients with pancreatic cancer were at advanced stage when diagnosed and most patients died within 1 year after receiving a confirmed diagnosis (3).

Classical Wnt/ $\beta$-catenin signaling pathway is one of signal transduction pathways widely studied in recent years. When Wnt pathway is activated abnormally, $\beta$-catenin is separated from compounds constituted of axin/APC and GSK-3 $\beta$,

Correspondence to: Dr Di Kong, Department of Oncology, Tianjin Nankai Hospital, 6 Changjiang Road, Nankai, Tianjin 300100, P.R. China

E-mail: dikongtj@163.com

Key words: miRNA-27a, human pancreatic cancer cell, Wnt, $\beta$-catenin accumulate and is transferred into cells, followed by the combination with TCF/LEF (4). Expression of downstream target genes as c-Myc and cyclin D1 are regulated to control cell cycle progress (5). Abnormally activated Wnt/ $\beta$-catenin signaling pathway plays an essential role in occurrence and metastasis of PAD. Activation of $\mathrm{Wnt} / \beta$-catenin signaling pathway at different levels has been shown in cell lines of pancreatic cancer and animal models of pancreatic cancer (6). Through blocking Wnt/ $\beta$-catenin signaling pathway, cell proliferation can be inhibited and cell apoptosis promoted (6).

MicroRNA (miRNA) is a class of widely distributed non-coding small RNA, which is the single-strand small molecule RNA about 19-23 nucleotides in length at maturation stage (7). Mature miRNA inhibits target mRNA translation through complementary base pairing with 3'-untranslated region (UTR), 5'-UTR and coding domain of target mRNA (7). In this way, it can regulate target gene expression at post-transcription level (8). Bioinformatics research has indicated that single miRNA molecule can bond with hundreds of target mRNAs with diverse functions, thus exerting the regulatory function (9). Moreover, it is involved in almost all pathological and physiological activities in mammals, such as individual development, tissue differentiation, cell apoptosis and energy metabolism (10). In addition, it is closely associated with the genesis and development of numerous diseases (10). Plenty of recent studies have reported that serum/plasma miRNA expression profile can effectively distinguish tumor patients from healthy individuals (11). Therefore, the aims of this study were to examine the effect of miRNA-27a on cell growth and induction of apoptosis of human pancreatic cancer cells.

\section{Materials and methods}

Patients samples. Six pancreatic cancer patients and six healthy volunteers were collected from Department of Oncology, Tianjin Nankai Hospital. Serum of pancreatic cancer patients and healthy volunteers were collected and centrifuged at $1,000 \mathrm{~g}$ for $20 \mathrm{~min}$ at $4^{\circ} \mathrm{C}$, and saved at $-80^{\circ} \mathrm{C}$.

RNA extraction and quantitative real-time polymerase chain reaction. Total RNA was extracted with miRNeasy Mini kit (Qiagen Co., Hilden, Germany). cDNA was reverse transcribed using the TransScript First-Strand cDNA Synthesis SuperMix (Invitrogen). qRT-PCR was performed by ABI 7500 real-time 
PCR instrument (ABI Co., Oyster Bay, NY, USA) using FastStart Universal STBR Green Master (ROX) (Roche, Basel, Switzerland). miR-27a: 5'-GGCTTAGCTGCTTGTGA GCA-3'; reverse primer of miR-27a: 5'-GCGGAACTTAG CCACTGTGA-3'. U6-F: 5'-CTCGCTTCGGCAGCACA-3', U6-R: 5'-AACGCTTCACGAATTTGCGT-3'.

Chemicals. Dulbecco's modified Eagle's medium (DMEM) and fetal bovine serum (FBS) were obtained from Gibco BRL (Grand Island, NY, USA). Penicillin and streptomycin were obtained from Invitrogen (Carlsbad, CA, USA). Guava Nexin ${ }^{\mathrm{TM}}$ kit was obtained from Roche (Indianapolis, IN, USA). Caspase-Glo assays were obtained from Provo McGonagall (Beijing) Biological Technology Co., Ltd. (Beijing, China).

Cell culture and luciferase assay. Human pancreatic cancer cell PANC-1 cells were obtained and were maintained in DMEM medium containing penicillin $(50 \mathrm{U} / \mathrm{ml})$, streptomycin $(50 \mathrm{U} / \mathrm{ml})$ and $10 \%$ FBS in an incubator with a humidified atmosphere of $5 \% \mathrm{CO}_{2}$ at $37^{\circ} \mathrm{C}$. Cell viability of PANC-1 cells was determined using MTT assay (Sigma-Aldrich, St. Louis, MO, USA). miRNA-27a, anti-miRNA-27a and negative mimics were purchased from Sangon Biotech Co., Ltd. (Shanghai, China). Cell was transfected using Lipo 3000 reagent (Thermo Fisher Scientific, Inc., Waltham, MA, USA).

Cell viability assay and cytotoxicity. PANC-1 cells were seeded in 96-well plates and transfected with miRNA-27a, anti-miRNA-27a and negative mimics for 0, 24, 48 and $72 \mathrm{~h}$. MTT assay $(10 \mu \mathrm{l})(5 \mu \mathrm{g} / \mathrm{ml})$ was added to each well and incubated for $4 \mathrm{~h}$ at $37^{\circ} \mathrm{C}$ in the dark. DMSO assay $(200 \mu \mathrm{l})$ was dissolved into each well and shaked for $20 \mathrm{~min}$. In brief, LDH assay induced nicotinamide adenine dinucleotide, which modified tetrazolium dye to a soluble, colored formazan derivative. Plate microreader (Perkin-Elmer, San Diego, CA, USA) was used to detect cell absorbance at $490 \mathrm{~nm}$.

Determination of apoptosis.PANC-1 cells were seeded in 6-well plates and transfected with miRNA-27a, anti-miRNA-27a and negative mimics for $48 \mathrm{~h}$. Apoptosis of PANC-1 cells were analyzed using the Guava Nexin ${ }^{\mathrm{TM}}$ kit by flow cytometry (Roche, Indianapolis, IN, USA). Annexin V-P $(10 \mu \mathrm{l})$ was added into PANC-1 cells and incubated for $30 \mathrm{~min}$ in darkness. PI $(10 \mu \mathrm{l})$ was added into PANC-1 cells and incubated for $30 \mathrm{~min}$ in darkness. Flow cytometry (BD Biosciences) was used to detect apoptosis.

Measurement of caspase-3/9 activity. PANC-1 cells were seeded in 6-well plates and transfected with miRNA-27a, anti-miRNA-27a and negative mimics for $48 \mathrm{~h}$. Appropriate Caspase-Glo reagent $(100 \mu \mathrm{l})$ was added into each well and incubated for $1 \mathrm{~h}$ at room temperature. Plate microreader (Perkin-Elmer) was used to detect caspase-3/9 activity absorbance at $405 \mathrm{~nm}$.

Western blot analysis. PANC-1 cells were seeded in 6-well plates and transfected with miRNA-27a, anti-miRNA-27a and negative mimics for $48 \mathrm{~h}$. The cells were then cultivated by collecting the supernatant and lysed on ice in a buffer (Beyotime Biotech, Nanjing China). Protein content was quantitated using

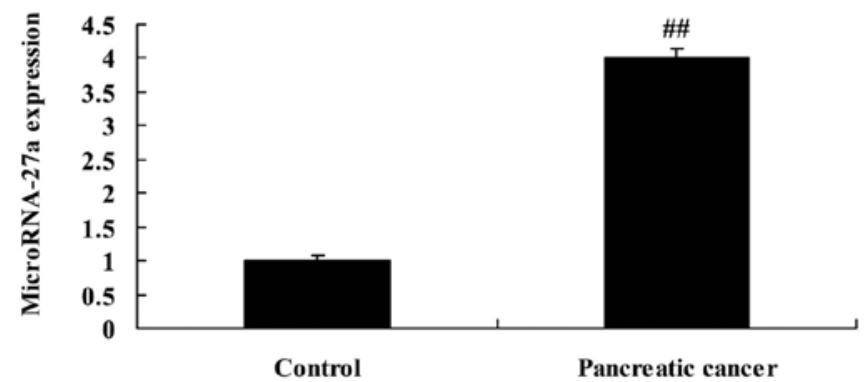

Figure 1. miRNA-27a expression of pancreatic cancer. Control, normal control group. Pancreatic cancer, pancreatic cancer patients group. ${ }^{\# \#} \mathrm{p}<0.01$ compared with control group.

BCA protein assay kit (Pierce, Rockford, IL, USA). A protein sample of $20 \mu \mathrm{g}$ was resolved on $12 \%$ SDS-PAGE gel and transferred onto polyvinylidene difluoride membrane. Then, the membrane was washed three times with $0.1 \%$ (v/v) Tween-20 in Tris-buffered saline solution (TTBS; Biosharp, St. Louis, MO, USA). The membrane was incubated with anti-PAK1 (diluted 1:500; Santa Cruz Biotechnology, Santa Cruz, CA, USA), anti-phosphorylation of ERK (p-ERK, diluted 1:1,000; Santa Cruz Biotechnology), anti-Wnt (diluted 1:1,000; Santa Cruz Biotechnology), anti- $\beta$-catenin (diluted 1:1,000; Santa Cruz Biotechnology) and GAPDH (Beyotime Biotech) at $4^{\circ} \mathrm{C}$ for $6 \mathrm{~h}$. The membrane was incubated the secondary alkaline phosphatase conjugated goat anti-mouse IgG (diluted 1:500 in TBS; Beyotime Biotech) for $2 \mathrm{~h}$.

Statistical analysis. The data are expressed as the mean \pm standard deviation analyzed using SPSS version 17.0 statistical software (SPSS, Inc., Chicago, IL, USA). An analysis of variance was performed where appropriate, and the StudentNewman-Keuls method was used for pairwise comparison. $\mathrm{P}<0.05$ was considered to indicate a statistically significant difference.

\section{Results}

miRNA-27a expression of pancreatic cancer. To determine whether the expression of miRNA-27a altered pancreatic cancer, we extracted serum to measure miRNA-27a expression. As shown in Fig. 1, miRNA-27a expression in serum of pancreatic cancer was upregulated, compared with normal group.

Upregulation of miRNA-27a expression induces cell growth and migration of pancreatic cancer. To investigate the specific role of miRNA-27a in regulation of cell growth of pancreatic cancer, miRNA-27a was upregulated using miRNA-27a mimics. As shown in Fig. 2A-D, miRNA-27a expression was significantly upregulated, upregulation of miRNA-27a expression significantly induced cell growth and migration of pancreatic cancer, compared with control group.

Upregulation of miRNA-27a expression inhibits apoptosis of pancreatic cancer. Apoptosis rate and caspase-3/9 activity were significantly inhibited by miRNA-27a upregulation of pancreatic cancer, compared with control group (Fig. 2E-H). 

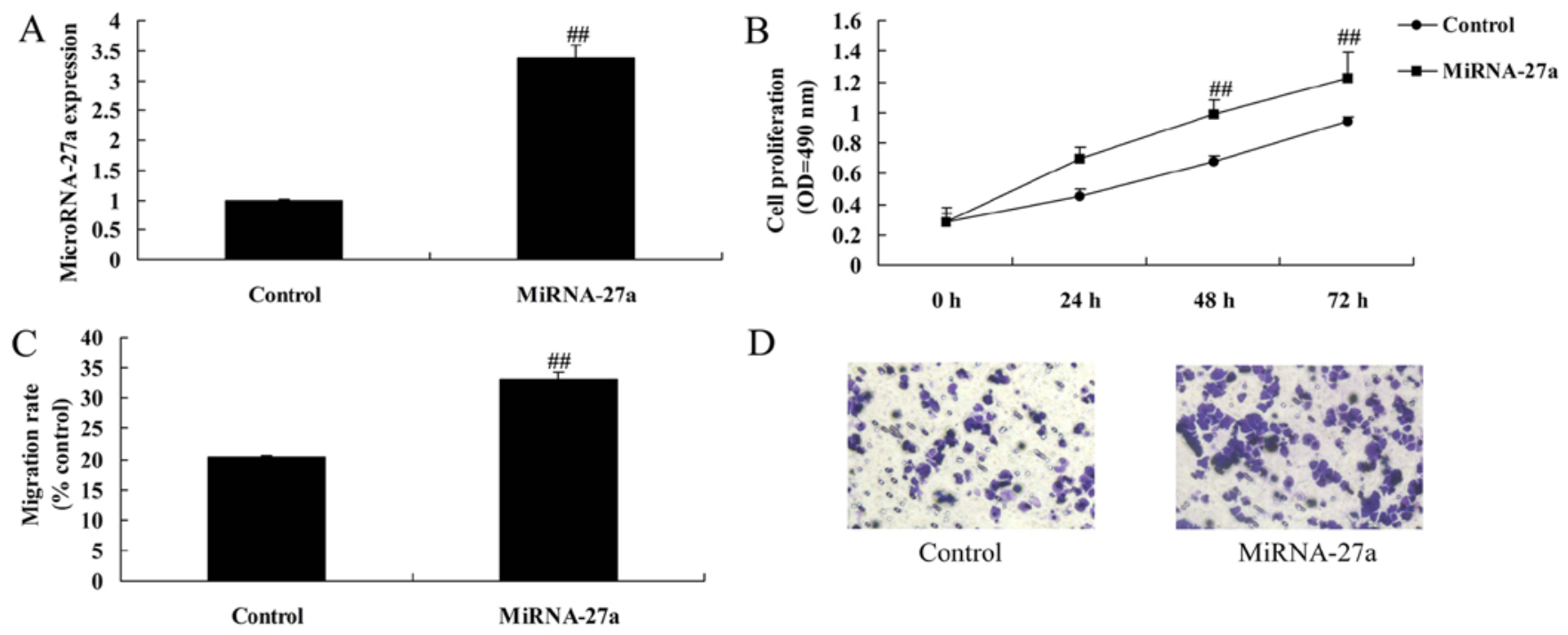

D
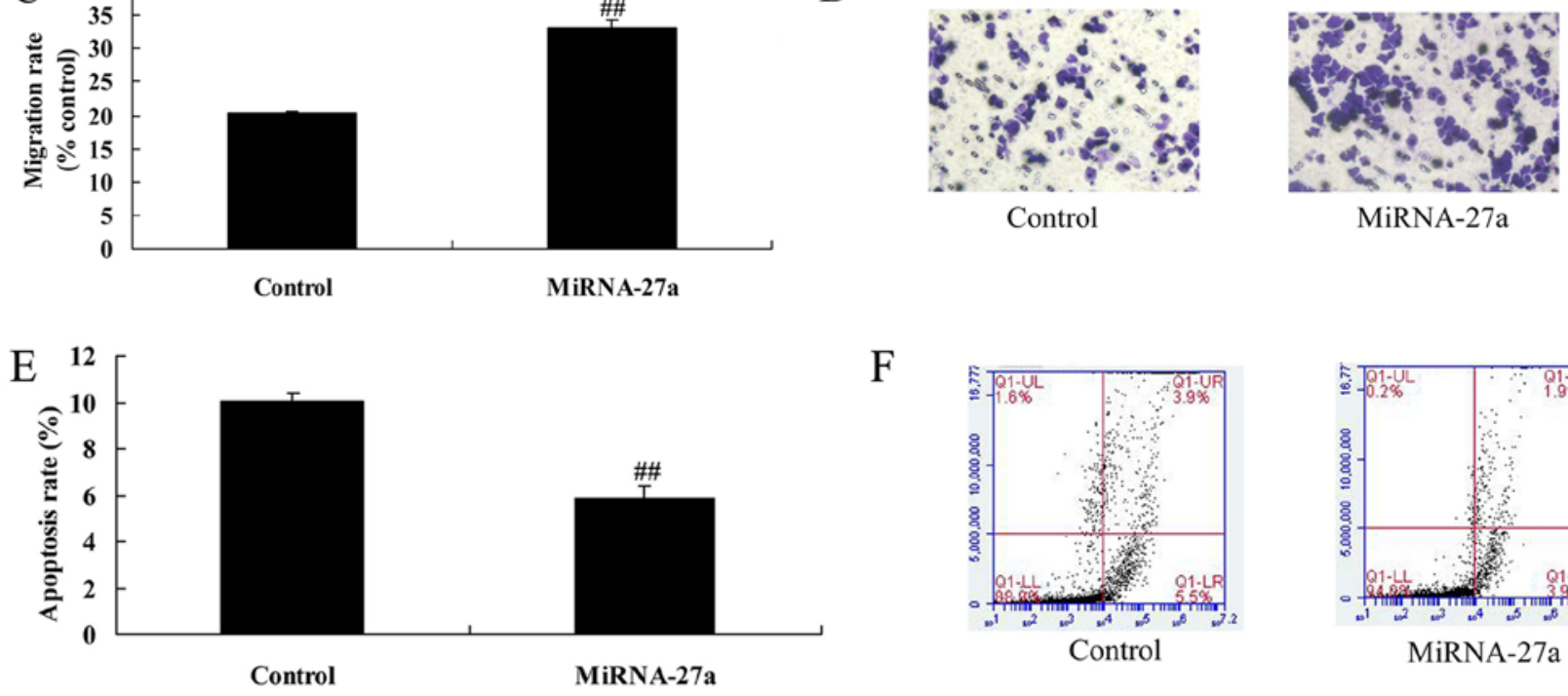

F
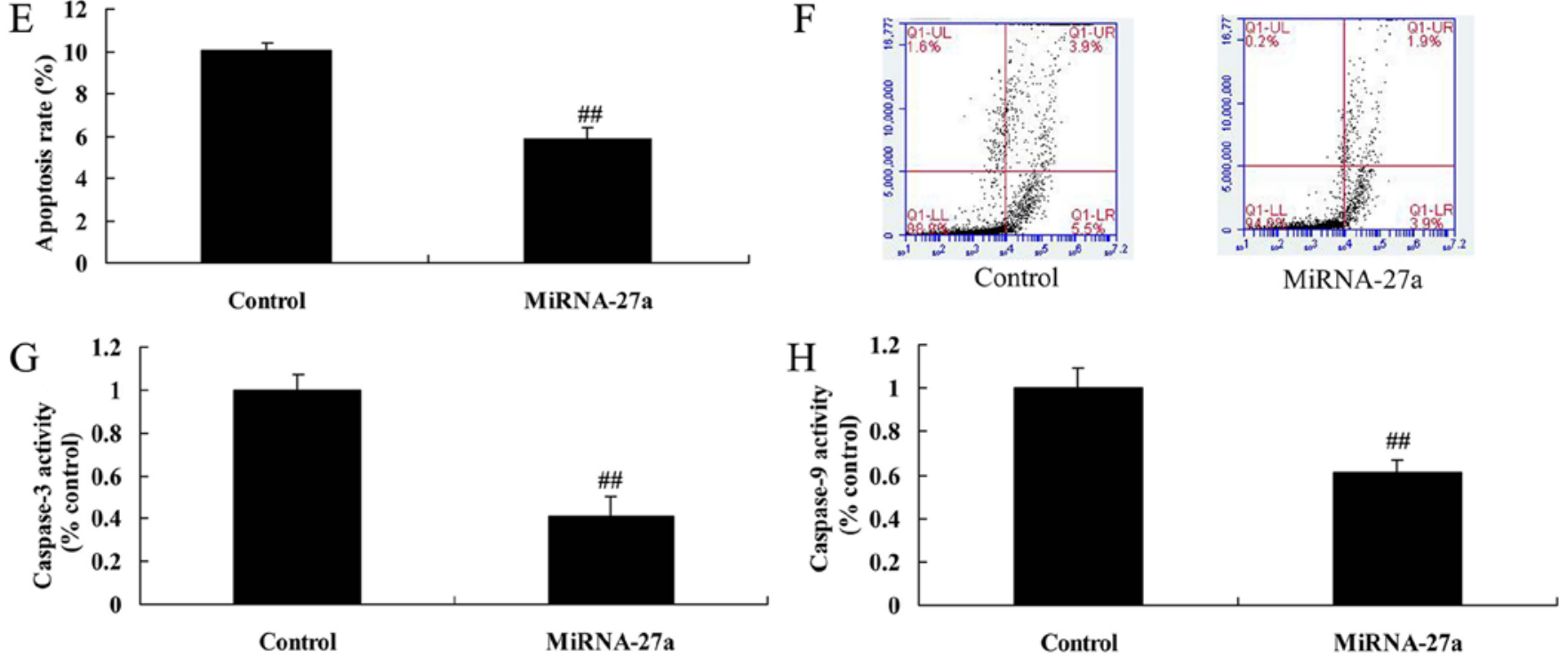

Figure 2. Upregulation of miRNA-27a expression induces cell growth, migration and apoptosis of pancreatic cancer. miRNA-27a expression (A), cell growth (B), migration rate (C and D), apoptosis rate (E and F), and caspase-3 (G) and caspase-9 activity (H). Control, negative control group; miRNA-27a, upregulation of miRNA-27a group. ${ }^{\#} \mathrm{p}<0.01$ compared with the control group.

Upregulation of miRNA-27a expression inhibits Bax and induced cyclin D1 protein of pancreatic cancer. The protein expression of Bax was significantly suppressed, cyclin D1 protein expression was significantly induced by miRNA-27a upregulation of pancreatic cancer, compared with control group (Fig. 3A-C).

Upregulation of miRNA-27a expression induces $W n t / \beta$-catenin pathway of pancreatic cancer. To assess the mechanism by which miRNA-27a induces apoptosis of PANC-1 cells, Wnt/ß-catenin pathway was analyzed. As shown in Fig. 3D-F, upregulation of miRNA-27a expression significantly induced Wnt/ $\beta$-catenin pathway of pancreatic cancer, compared with control group.

Downregulation of miRNA-27a expression reduces cell growth and migration of pancreatic cancer. Next, we reduced miRNA-27a expression using anti-miRNA-27a mimics. As shown in Fig. 4A-D, the downregulation of miRNA-27a expression significantly reduced cell growth and migration of pancreatic cancer, compared with the control group.

Downregulation of miRNA-27a expression induces apoptosis of pancreatic cancer. Fig. 4E-H showed that the downregulation of miRNA-27a expression significantly induced apoptosis and caspase-3/9 activity of pancreatic cancer, compared with control group.

Downregulation of miRNA-27a expression induces Bax and suppresses cyclin DI protein of pancreatic cancer. Downregulation of miRNA-27a expression significantly induced Bax protein expression and suppressed cyclin D1 protein expression of pancreatic cancer, compared with control group (Fig. 5A-C).

Downregulation of miRNA-27a expression suppressed Wnt/ $\beta$-catenin pathway of pancreatic cancer. To study the mechanism of miRNA-27a on apoptosis of pancreatic cancer, 

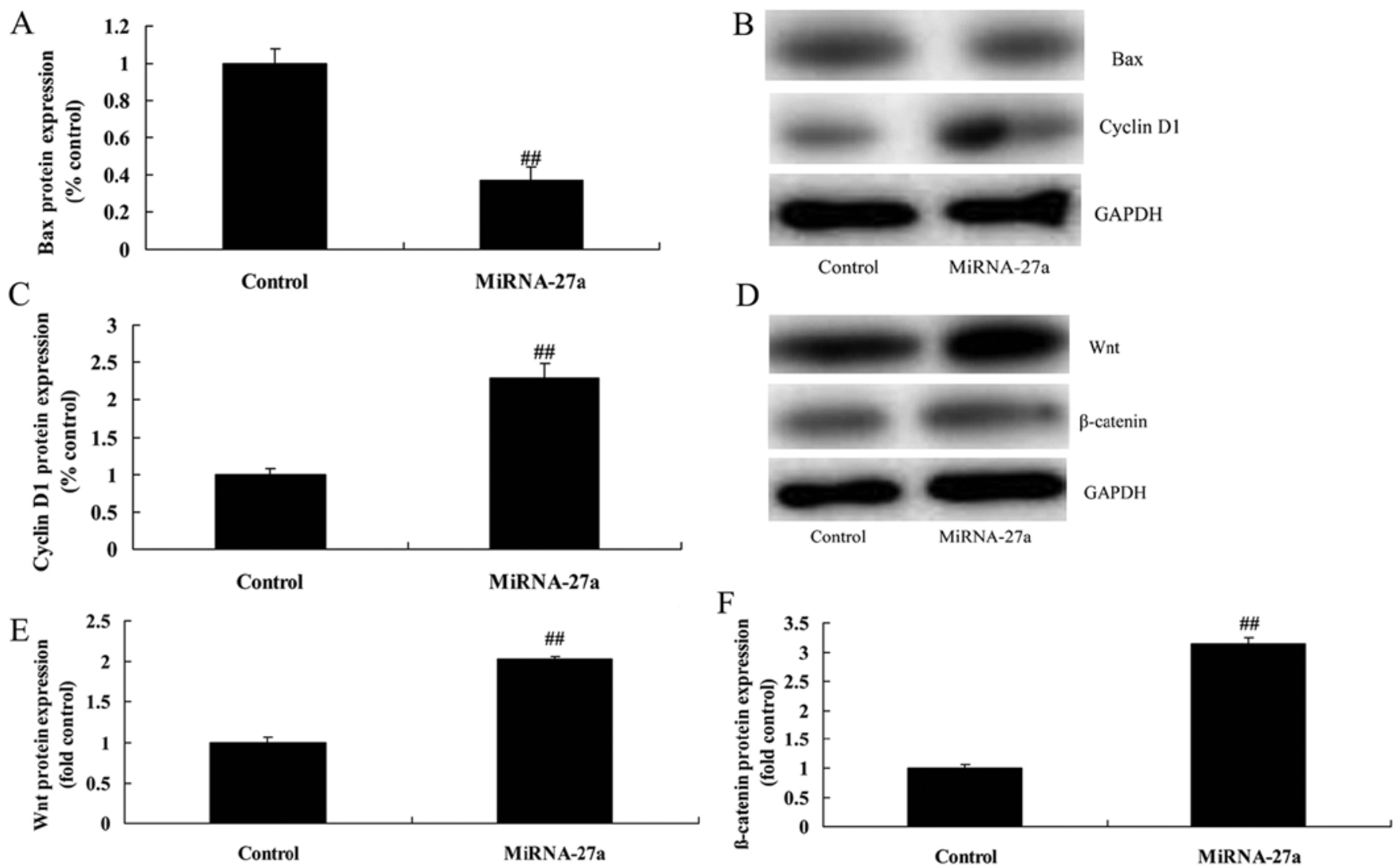

Figure 3. Upregulation of miRNA-27a expression affects Bax, cyclin D1, Wnt and $\beta$-catenin protein of pancreatic cancer. Bax and cyclin D1 protein expression (A and B), and western blot analysis for Bax and cyclin D1 protein expression (C). Western blot analysis for Wnt and $\beta$-catenin protein expression (D), and Wnt and $\beta$-catenin protein expression (E and F). Control, negative control group; miRNA-27a, upregulation of miRNA-27a group. ${ }^{\# \# p<0.01 ~ c o m p a r e d ~ w i t h ~}$ control group.

Wnt/ $\beta$-catenin pathway was measured using western blot analysis. Wnt/ $\beta$-catenin pathway of pancreatic cancer was significantly suppressed by miRNA-27a downregulation, compared with the control group (Fig. 5D-F).

The inhibition of Wnt/ $\beta$-catenin pathway suppresses Wnt/ $\beta$-catenin pathway of pancreatic cancer following anti-miRNA-27a. In order to strengthen the involvement of Wnt pathway in anti-miRNA-27a induced apoptosis, we next checked the levels of Wnt protein expression after Wnt inhibitor. As showed in Fig. 6A-C, Wnt inhibitor, Wnt-C59, $25 \mathrm{pM}$, for $48 \mathrm{~h}$, suppressed $\mathrm{Wnt} / \beta$-catenin pathway of pancreatic cancer following anti-miRNA-27a, compared with anti-miRNA-27a group.

The inhibition of Wnt/ $\beta$-catenin pathway increases the anticancer effects of anti-miRNA-27a on Bax and cyclin DI protein of human pancreatic cancer. The induction of Bax protein expression and suppression of cyclin D1 protein of human pancreatic cancer following anti-miRNA-27a were promoted by inhibition of Wnt/ $\beta$-catenin pathway, compared with anti-miRNA-27a group (Fig. 6D-F).

The inhibition of Wnt/ $\beta$-catenin pathway increases the anticancer effects of anti-miRNA-27a on human pancreatic cancer cells. Compared with anti-miRNA-27a group, the inhibition of $\mathrm{Wnt} / \beta$-catenin pathway increased the anticancer effects of
anti-miRNA-27a on the inhibition of human pancreatic cancer cell growth and migration of pancreatic cancer (Fig. 7A-D).

The inhibition of Wnt/ $\beta$-catenin pathway increases the anticancer effects of anti-miRNA-27a on apoptosis of human pancreatic cancer. The promotion of apoptosis rate and caspase-3/9 activity of human pancreatic cancer following anti-miRNA-27a was increased by inhibition of Wnt/ $\beta$-catenin pathway, compared with anti-miRNA-27a group (Fig. 7D-G).

The inhibition of Wnt/ $\beta$-catenin pathway decreases the effects of miRNA-27a on human pancreatic cancer cell. Moreover, we found that the inhibition of Wnt $/ \beta$-catenin pathway suppressed the effects of anti-miRNA-27a on the induction of Wnt/ $\beta$ catenin pathway in human pancreatic cancer cells, compared with miRNA-27a group (Fig. 8A-C). However, the inhibition of Wnt/B-catenin pathway induced Bax protein expression and suppressed cyclin D1 protein expression in human pancreatic cancer cells, compared with miRNA-27a group (Fig. 8D-F).

The inhibition of Wnt/ $\beta$-catenin pathway decreases the effects of miRNA-27a on apoptosis of human pancreatic cancer. The inhibition of Wnt/ $\beta$-catenin pathway decreased the effects of miRNA-27a on the promotion of cell growth and migration of pancreatic cancer, and the inhibition of apoptosis and caspase-3/9 activity of human pancreatic cancer, compared with miRNA-27a group (Fig. 9). 
A

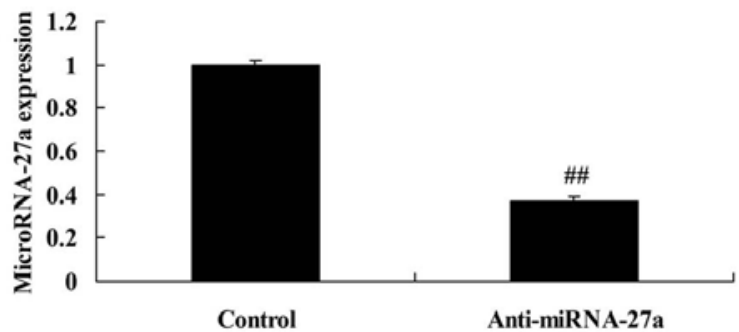

C

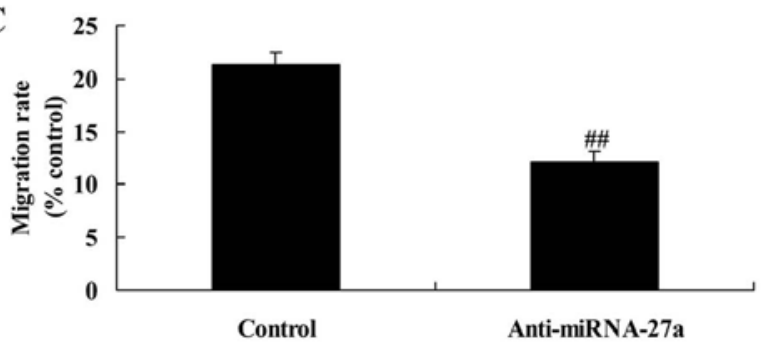

E

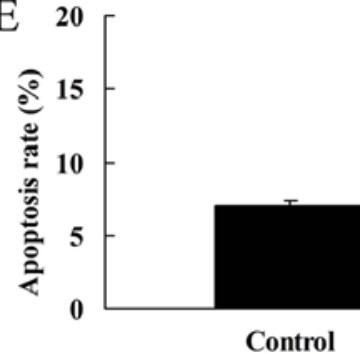

G

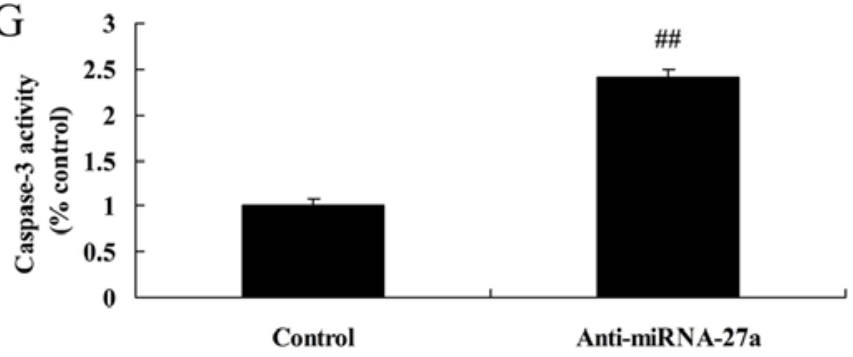

B

D
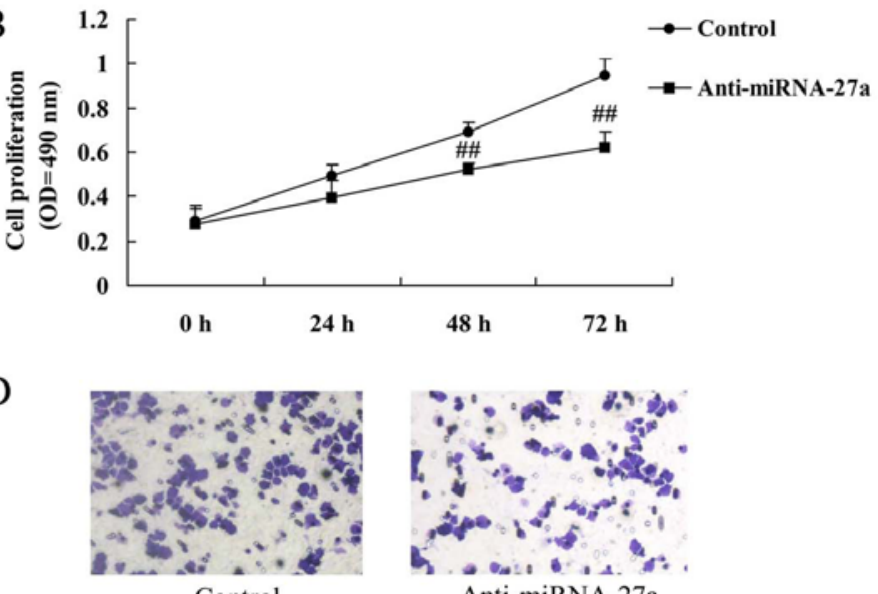

Control

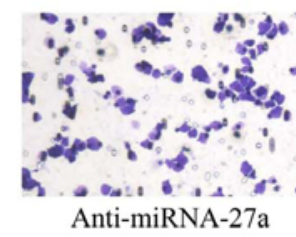

F
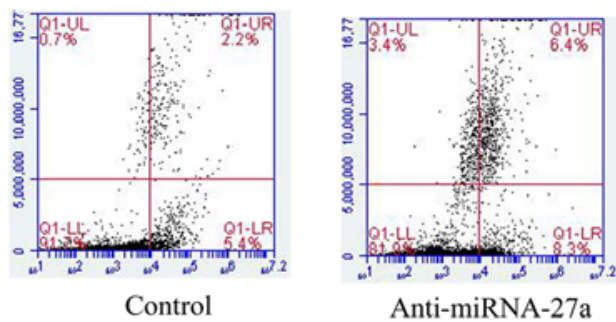

$\mathrm{H}$

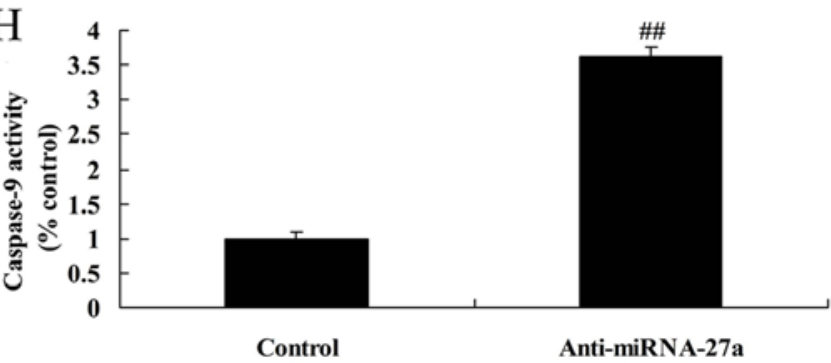

Figure 4. Downregulation of miRNA-27a expression reduced cell growth, migration and apoptosis of pancreatic cancer. miRNA-27a expression (A), cell growth (B), migration rate (C and D), apoptosis rate (E and F), and caspase-3 (G) and caspase-9 activity (H). Control, negative control group. Anti-miRNA-27a, downregulation of miRNA-27a group. ${ }^{\# \#} \mathrm{p}<0.01$ compared with control group.

\section{Discussion}

With a rapid course, the onset of pancreatic cancer is hidden. Early diagnostic methods have not been identified. When making definite diagnosis, metastasis has already occurred. It is low in resectability with poor prognosis (1). Statistics from American Cancer Society show that in 2006, 33,730 new cases of pancreatic cancer occurred in US and $\sim 32,300$ people died of it (2). Its morbidity rate has not obviously changed for decades. Its death rate ranks the fourth among all malignant cancers. Herein we found that miRNA-27a expression in serum of pancreatic cancer was upregulated, compared with normal group. In this study, we only measured miRNA-27a expression serum of pancreatic cancer, which may still be insufficient, and we will analyze the expression of miR-27 in cancer cells from cell line or human tissue in further study.

Molecular diagnostics is a novel clinical diagnostic method developed in recent years, targeting the detection of DNA, RNA and protein (12). miRNA has attracted extensive attention from specialists and scholars in recent years (13). Altogether 1,048 kinds of human miRNAs have been discovered at present, which differ in their functions to regulate expression (13). Moreover, abnormal miRNA expression is suggested to be the early event in pancreatic cancer, which can reflect the genesis, development and pathological classification of pancreatic cancer (14). This makes it possible to use miRNA as the novel biomarker in the early diagnosis of pancreatic cancer (15). Our study showed that anti-miRNA-27a inhibited cell growth and apoptosis in pancreatic cancer cells. Li and Luo ascertained that miR-27a-3p promotes nasopharyngeal carcinoma cell proliferation and migration (16).

PAK1 has high expression in various tumor cells, and also participates in events such as genetic transcription, cell division and cell movement. We have little knowledge on the relationship between PAK and Wnt signaling pathway (17). Current studies are being concentrated on the interaction between PAK1 and proteins in Wnt signaling pathway. Other connection between PAK1 and Wnt signal is that Wnt1 can 

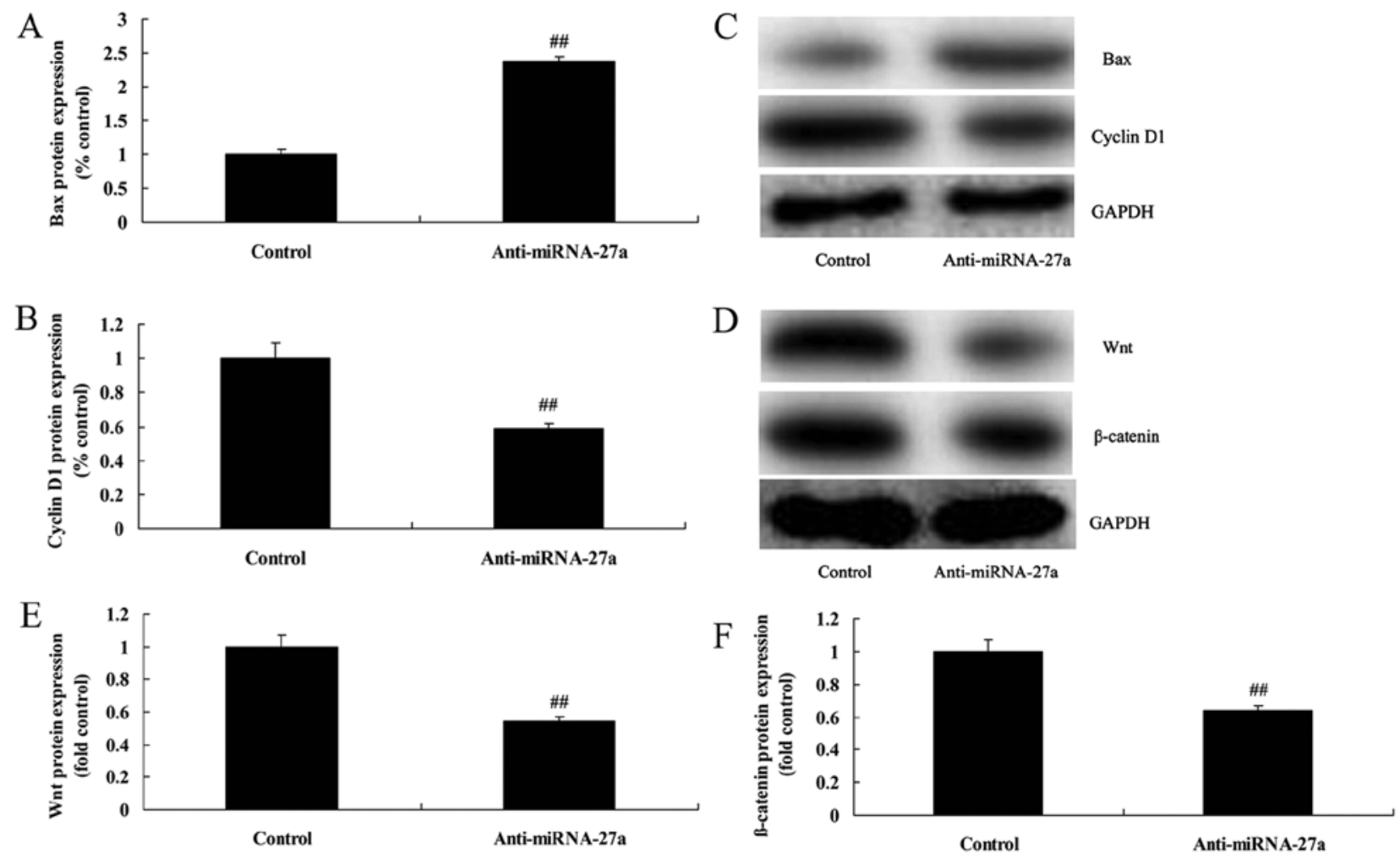

Figure 5. Downregulation of miRNA-27a expression induces Bax and suppresses cyclin D1 protein of pancreatic cancer. Bax and cyclin D1 protein expression (A and B), and western blot analysis for Bax and cyclin D1 protein expression (C). Western blot analysis for Wnt and $\beta$-catenin protein expression (D), and Wnt and $\beta$-catenin protein expression (E and F). Control, negative control group. Anti-miRNA-27a, downregulation of miRNA-27a group. ${ }^{\# \#}$ p $<0.01$ compared with control group.
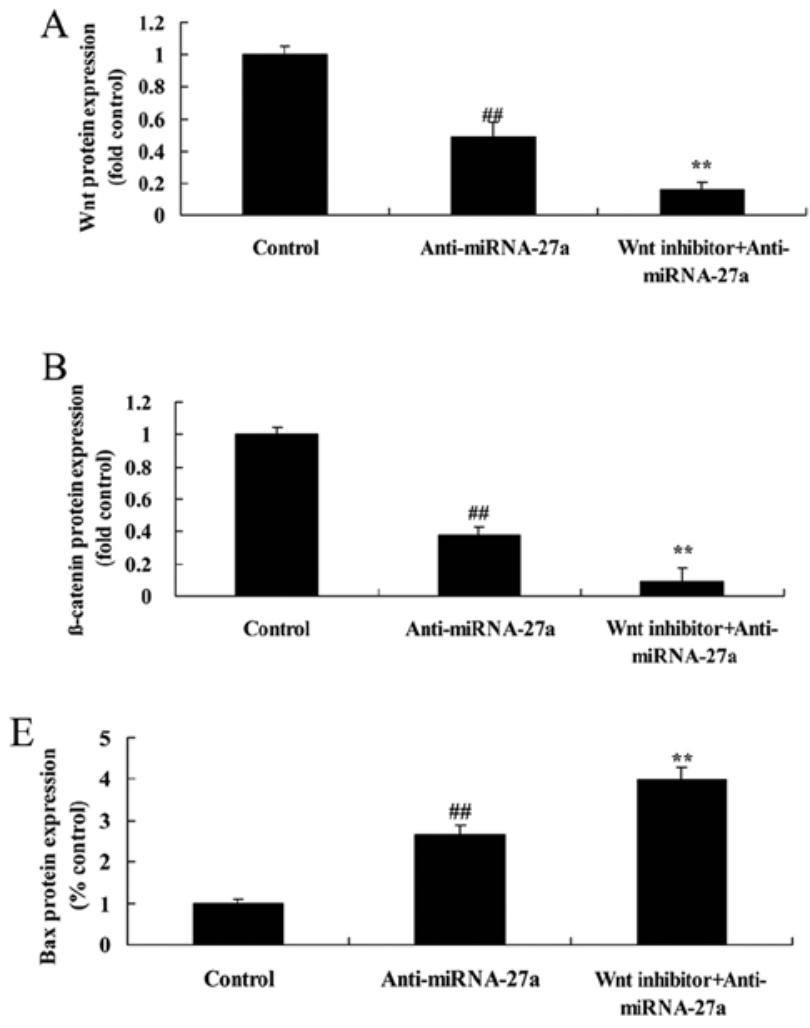

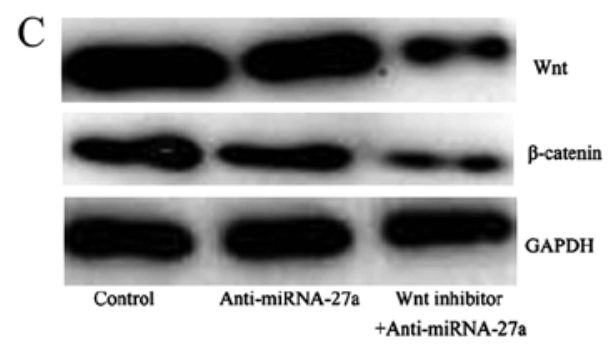

$\mathrm{D}$
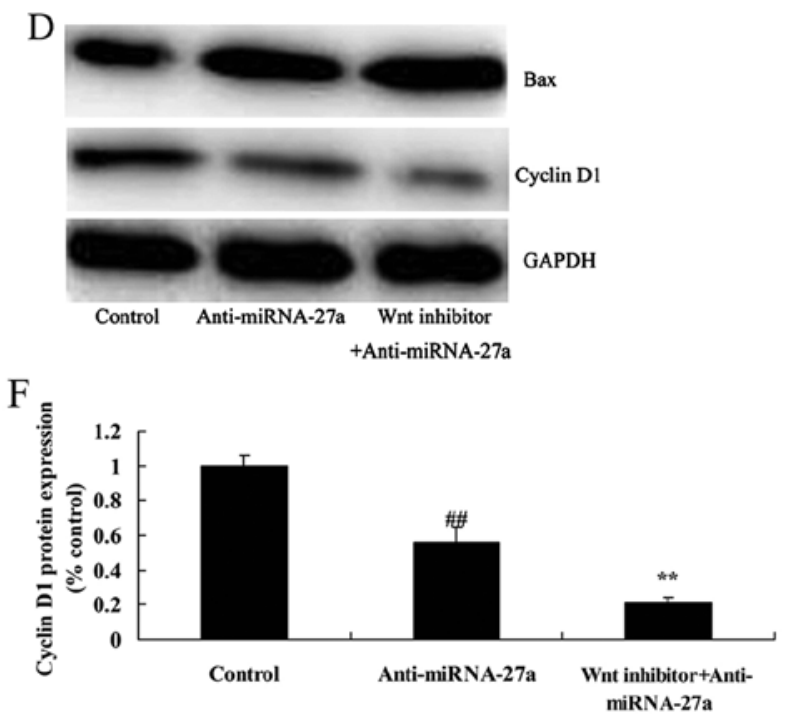

Figure 6. The inhibition of Wnt/ $\beta$-catenin pathway suppresses Wnt/ $\beta$-catenin pathway of pancreatic cancer following anti-miRNA-27a. Wnt and $\beta$-catenin protein expression (A and B), and western blot analysis for Wnt and $\beta$-catenin protein expression (C). Bax and cyclin D1 protein expression (D and E), and western blot analysis for Bax and cyclin D1 protein expression (F). Control, negative control group. Anti-miRNA-27a, downregulation of miRNA-27a group. Wnt inhibitor+Anti-miRNA-27a, Wnt inhibitor and downregulation of miRNA-27a group. ${ }^{\# \#}$ p $<0.01$ compared with control group; ${ }^{* *} \mathrm{p}<0.01$ compared with anti-miRNA-27a group. 

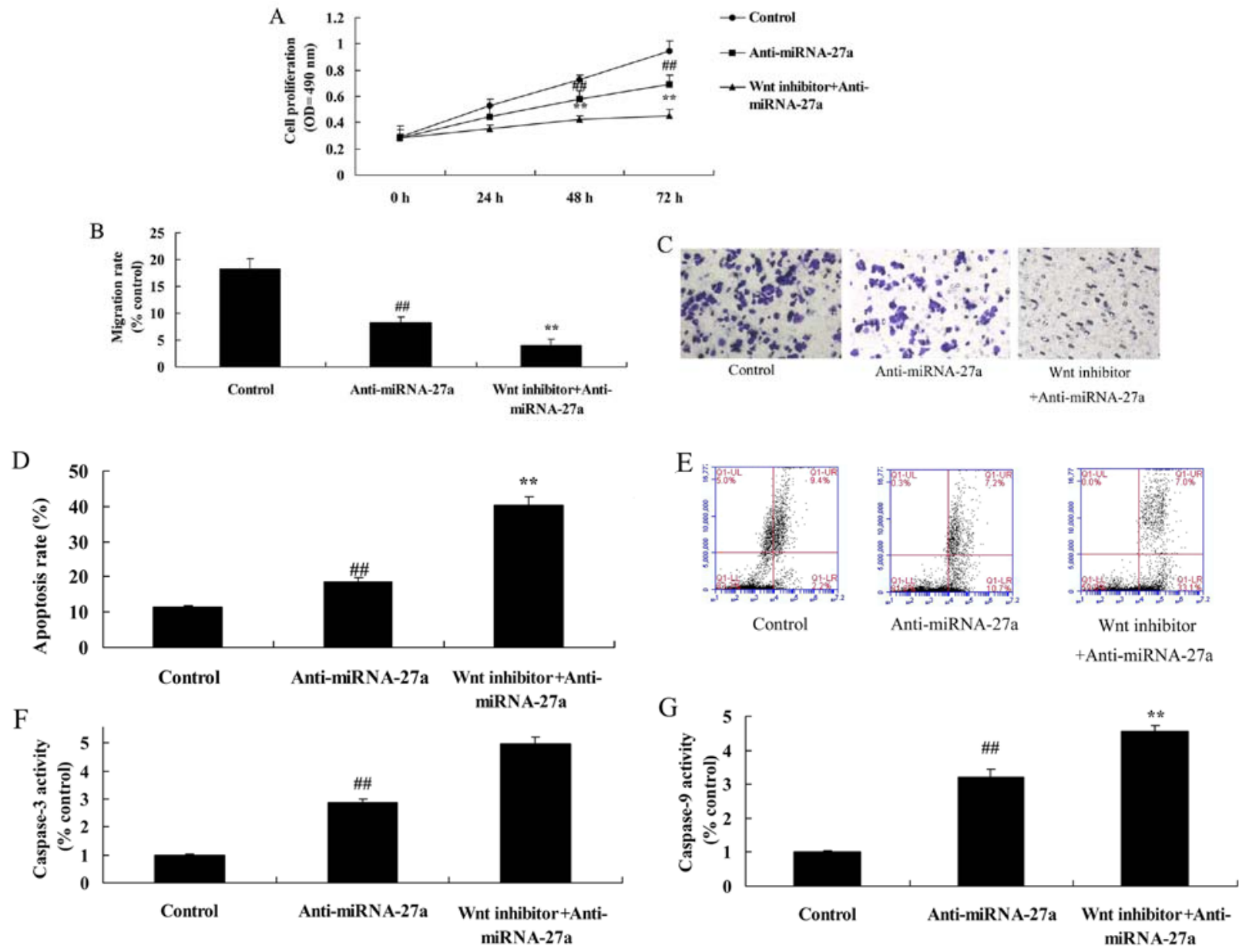

Figure 7. The inhibition of Wnt/ $\beta$-catenin pathway increased the anticancer effects of anti-miRNA-27a on human pancreatic cancer cells. Cell growth (A), migration rate (B and C), apoptosis rate (D and E), and caspase-3 (F) and caspase-9 activity (G). Control, negative control group. Anti-miRNA-27a, downregulation of miRNA-27a group. Wnt inhibitor+Anti-miRNA-27a, Wnt inhibitor and downregulation of miRNA-27a group. ${ }^{\#}$ p $<0.01$ compared with control group; ${ }^{* *} \mathrm{p}<0.01$ compared with anti-miRNA-27a group.

A

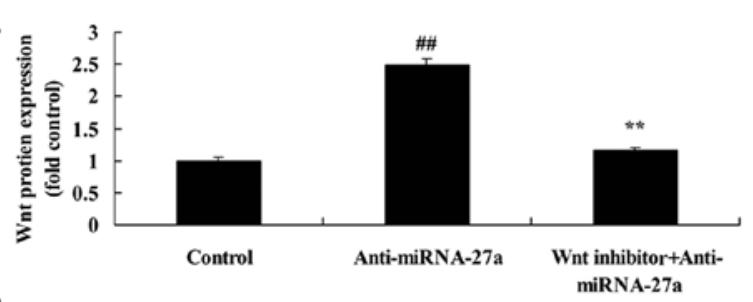

B

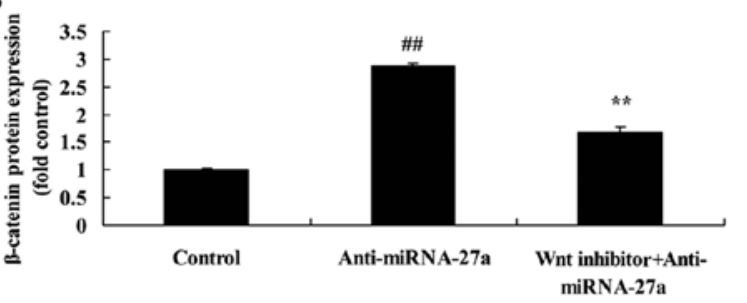

$\mathrm{E}$

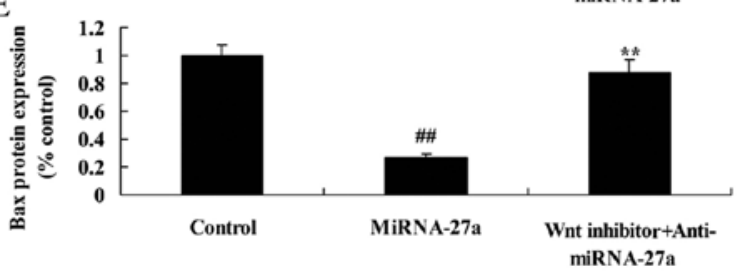

C

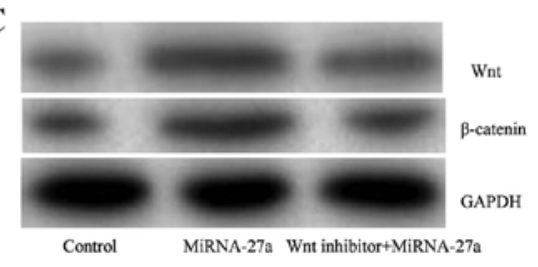

$\mathrm{D}$
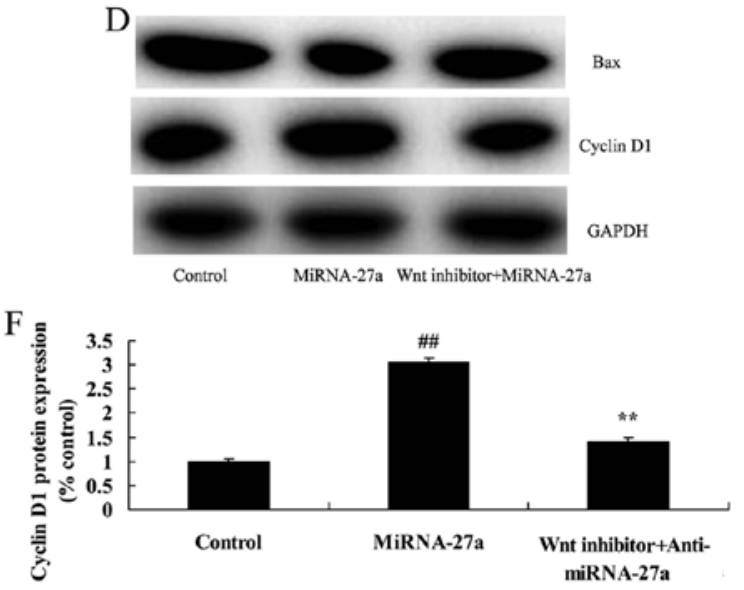

Figure 8. The inhibition of Wnt/ $\beta$-catenin pathway decreases the effects of miRNA-27a on human pancreatic cancer cells. Wnt and $\beta$-catenin protein expression (A and B), and western blot analysis for Wnt and $\beta$-catenin protein expression (C). Bax and cyclin D1 protein expression (D and E), and western blot analysis for Bax and cyclin D1 protein expression (F). Control, negative control group. miRNA-27a, upregulation of miRNA-27a group. Wnt inhibitor+ miRNA-27a, Wnt inhibitor and upregulation of miRNA-27a group. ${ }^{\# \#} \mathrm{p}<0.01$ compared with control group, ${ }^{* *} \mathrm{p}<0.01$ compared with anti-miRNA-27a group. 

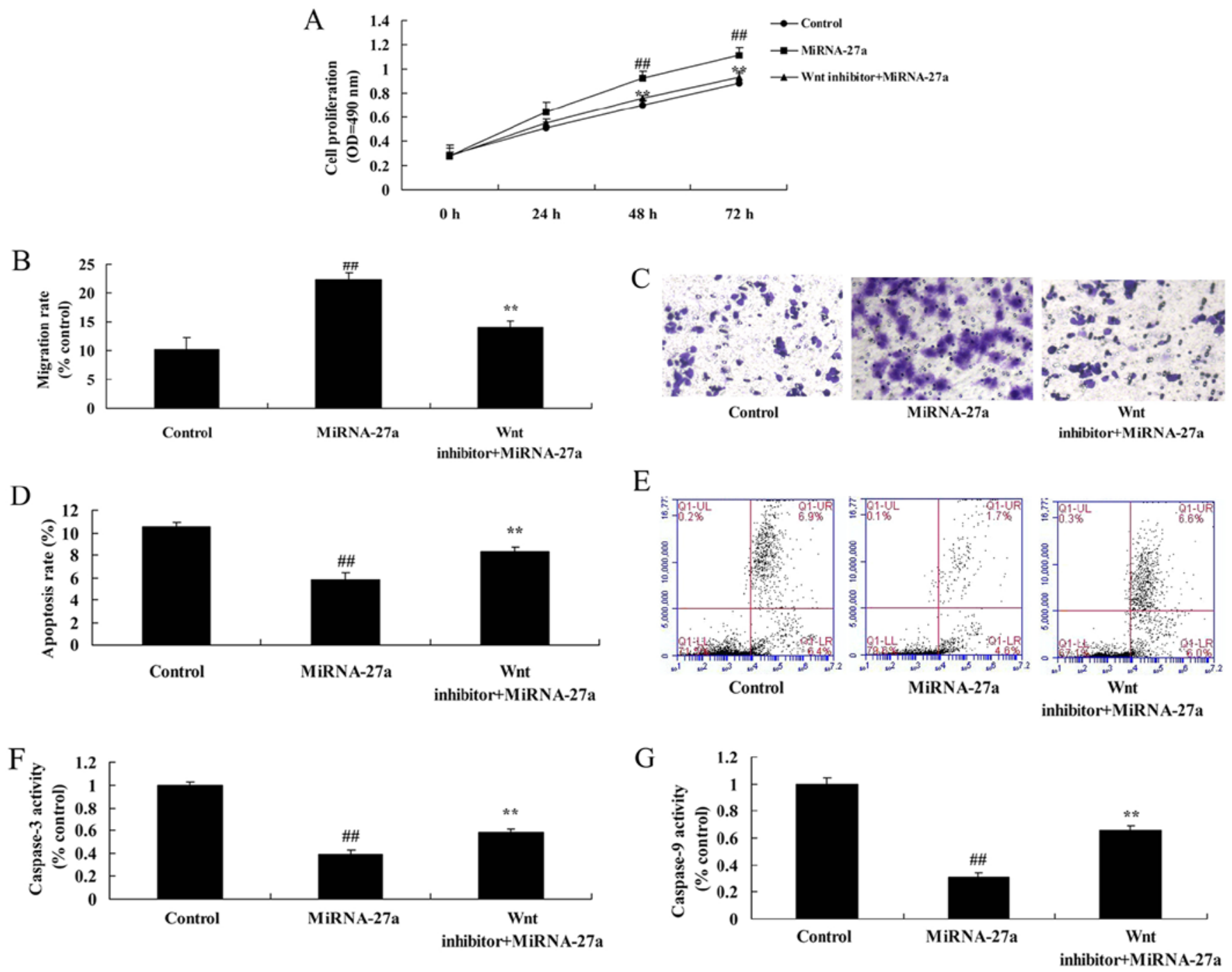

Figure 9. The inhibition of Wnt/ $\beta$-catenin pathway decreases the effects of miRNA-27a on apoptosis of human pancreatic cancer. Cell growth (A), migration rate (B and C), apoptosis rate (D and E), and caspase-3 (F) and caspase-9 activity (G). Control, negative control group. miRNA-27a, upregulation of miRNA-27a group. Wnt inhibitor+ miRNA-27a, Wnt inhibitor and upregulation of miRNA-27a group. ${ }^{\# \#} \mathrm{p}<0.01$ compared with control group; ${ }^{* *} \mathrm{p}<0.01$ compared with anti-miRNA-27a group.

activate PAK1 by inducing expression of WRCH1. The association between PAK1 and Wnt signal is close. It is reported that silent PAK1 would inhibit the accumulation of $\beta$-catenin stimulated by insulin. Moreover, reduction of phosphorylation levels at $\beta$-catenin S675 locus was also observed (18). In the present study, anti-miR-27a significantly suppressed PAK1 pathway of PANC-1 cells. Wang et al identified that miR-27a regulates Wnt/ $\beta$-catenin signaling through targeting SFRP1 in glioma (19). However, we only used Wnt-C59 together with anti-miRNA-27a in this study, which may be insufficient and we will execute miRNA-27a together with Wnt-C59 to examine whether a reverse effect existed.

Wnt signaling pathway plays an important role in embryonic development and tumorigenesis and $\beta$-catenin is a key component in signal processing of Wnt (5). c-Myc functions as the second target in Wnt signaling pathway. After shifting into cell nucleus, $\beta$-catenin reacts with transcription factors in Tcf/Lef family (20). $\beta$-catenin/Tcf complex activates target genes such as c-Myc, c-jun and cyclin D1 (5). Furthermore, it causes abnormal cell proliferation and differentiation, leading to the occurrence of tumors. In our study we found that anticancer effect of anti-miR-27a suppresses Wnt/ $\beta$-catenin pathway of PANC-1 cells. Wu et al showed that miR-27a could promote the proliferation and invasion of human gastric cancer via Wnt/ $\beta$-catenin signaling pathway (21). Wnt/ $\beta$-catenin pathway is complicated, we only checked the $\beta$-catenin signaling pathway and we will explore more signaling pathways (such as Akt, GSK-3) for miR-27a/Wnt in a further study.

In conclusion, we demonstrated that the miRNA-27a promotes the cell proliferation and inhibited apoptosis of human pancreatic cancer cell by activation of Wnt/ $\beta$-catenin pathway. These data suggest that miRNA-27a may represent a promising new index in currently human pancreatic cancer.

\section{References}

1. Cosentino M, Colombo C, Mauri M, Ferrari M, Corbetta S, Marino F, Bono G and Lecchini S: Expression of apoptosisrelated proteins and of mRNA for dopaminergic receptors in peripheral blood mononuclear cells from patients with Alzheimer disease. Alzheimer Dis Assoc Disord 23: 88-90, 2009. 
2. Moneim AE: Oxidant/antioxidant imbalance and the risk of Alzheimer's disease. Curr Alzheimer Res 12: 335-349, 2015.

3. Weinstein JD, Gonzalez ER, Egleton RD and Hunt DA: A paradigm shift for evaluating pharmacotherapy for Alzheimer's disease: The 10-patient screening protocol. Consult Pharm 28: 443-454, 2013.

4. Fan SH, Wang YY, Lu J, Zheng YL, Wu DM, Zhang ZF, Shan Q, Hu B, Li MQ and Cheng W: CERS2 suppresses tumor cell invasion and is associated with decreased V-ATPase and MMP-2/MMP-9 activities in breast cancer. J Cell Biochem 116 502-513, 2015.

5. Tang C, Chen L, Gu W, Du M, Li M, Chen Q and Li D: Cyclosporin A enhances the ability of trophoblasts to displace the activated human umbilical vein endothelial cell monolayers. Int J Clin Exp Pathol 6: 2441-2450, 2013.

6. Xie M, Hu A, Luo Y, Sun W, Hu X and Tang S: Interleukin-4 and melatonin ameliorate high glucose and interleukin- $1 \beta$ stimulated inflammatory reaction in human retinal endothelial cells and retinal pigment epithelial cells. Mol Vis 20: 921-928, 2014.

7. Zhang G, Liu D, Long G, Shi L, Qiu H, Hu G, Hu G and Liu S: Downregulation of microRNA-181d had suppressive effect on pancreatic cancer development through inverse regulation of KNAIN2. Tumour Biol 39: 1010428317698364, 2017.

8. Wang J, Guo XJ, Ding YM and Jiang JX: miR-1181 inhibits invasion and proliferation via STAT3 in pancreatic cancer. World J Gastroenterol 23: 1594-1601, 2017.

9. Lai X, Wang M, McElyea SD, Sherman S, House M and Korc M A microRNA signature in circulating exosomes is superior to exosomal glypican-1 levels for diagnosing pancreatic cancer. Cancer Lett 393: 86-93, 2017.

10. Xia SS, Zhang GJ, Liu ZL, Tian HP, He Y, Meng CY, Li LF, Wang ZW and Zhou T: MicroRNA-22 suppresses the growth, migration and invasion of colorectal cancer cells through a Spl negative feedback loop. Oncotarget 8: 36266-36278, 2017.

11. Liu F, Liu B, Qian J, Wu G, Li J and Ma Z: miR-153 enhances the therapeutic effect of gemcitabine by targeting Snail in pancreatic cancer. Acta Biochim Biophys Sin (Shanghai) 49: 520-529, 2017
12. Shigeyasu K, Toden S, Zumwalt TJ, Okugawa Y and Goel A: Emerging role of microRNAs as liquid biopsy biomarkers in gastrointestinal cancers. Clin Cancer Res 23: 2391-2399, 2017.

13. Wang BC and Ma J: Role of microRNAs in malignant glioma. Chin Med J (Engl) 128: 1238-1244, 2015.

14. Tung SL, Huang WC, Hsu FC, Yang ZP, Jang TH, Chang JW, Chuang CM, Lai CR and Wang LH: miRNA-34c-5p inhibits amphiregulin-induced ovarian cancer stemness and drug resistance via downregulation of the AREG-EGFR-ERK pathway. Oncogenesis 6: e326, 2017.

15. Li J, Su L, Gong YY, Ding ML, Hong SB, Yu S and Xiao HP. Downregulation of miR-139-5p contributes to the antiapoptotic effect of liraglutide on the diabetic rat pancreas and INS-1 cells by targeting IRS1. PLoS One 12: e0173576, 2017.

16. Li L and Luo Z: Dysregulated miR-27a-3p promotes nasopharyngeal carcinoma cell proliferation and migration by targeting Mapk10. Oncol Rep 37: 2679-2687, 2017.

17. Yarbro $\mathrm{CH}$ : International nursing and breast cancer. Breast $\mathrm{J} 9$ (Suppl 2): S98-S100, 2003.

18. Liu YF, Zhao Y, Wen XS and Dong QT: Advances in research on pharmacodynamics and chemical conversion of catalpol. Zhongguo Zhong Yao Za Zhi 32: 1128-1130, 2007 (In Chinese).

19. Wang K, Xie D, Xie J, Wan Y, Ma L, Qi X and Yang S: MiR-27a regulates Wnt/beta-catenin signaling through targeting SFRP1 in glioma. Neuroreport 26: 695-702, 2015.

20. Adams BD, Kasinski AL and Slack FJ: Aberrant regulation and function of microRNAs in cancer. Curr Biol 24: R762-R776, 2014.

21. Wu F, Li J, Guo N, Wang XH and Liao YQ: MiRNA-27a promotes the proliferation and invasion of human gastric cancer MGC803 cells by targeting SFRP1 via Wnt $/ \beta$-catenin signaling pathway. Am J Cancer Res 7: 405-416, 2017. 